\title{
Prostaglandin E2 inhibits IL-23 and IL-12 production by human monocytes through down-regulation of their common p40 subunit ${ }^{\text {is }}$
}

\author{
Khalid W. Kalim ${ }^{\mathrm{a}, \mathrm{b}}$, Marcus Groettrup a,b,c,* \\ a Division of Immunology, Department of Biology, University of Konstanz, D-78457 Konstanz, Germany \\ ${ }^{\mathrm{b}}$ Konstanz Research School Chemical Biology, University of Konstanz, D-78457 Konstanz, Germany \\ ${ }^{\mathrm{c}}$ Biotechnology Institute Thurgau at the University of Konstanz, CH-8280 Kreuzlingen, Switzerland
}

\begin{abstract}
A B S T R A C T
The heterodimeric cytokine IL-23 is important for the maintenance of Th17 cells, which are pivotal mediators of autoimmune diseases like rheumatoid arthritis, colitis, and multiple sclerosis. Prostaglandin E2 (PGE2) is a soluble regulator of inflammation that has both pro- and anti-inflammatory properties. PGE2 has been shown to elevate the IL-23 production by dendritic cells (DC). Monocytes are also producers of IL-23 but the effect of PGE2 on IL-23 production by human monocytes has hardly been investigated. We show here that PGE2 blocks the production of IL-23 by LPS-stimulated monocytes in an IL-10 and IL- $1 \beta$ independent manner. This effect was due to the down-regulation of the p40 subunit of IL-23 on mRNA and protein level. The p40 subunit is shared by IL-12 and, consistently, PGE2 also lowered the IL-12 production by monocytes. These effects of PGE2 were cAMP-dependent since the cAMP enhancer forskolin strongly reduced IL-23 and IL-12 production by monocytes. Taken together, PGE2 acts in an anti-inflammatory manner by lowering IL-23 production by monocytes while it has the opposite effect in DC. Our data may help to reconcile controversial point of views on the pro- and anti-inflammatory nature of PGE2 by making a strong case for a cell type-dependent function.
\end{abstract}

\section{Introduction}

Prostaglandins are small lipid molecules derived from arachidonic acid (AA) and are produced by the action of cyclooxygenases (COX-1 and COX-2) and prostaglandin synthases (Phipps et al., 1991). PGE2 is the most well studied prostaglandin and is involved in regulating many different fundamental biological functions both in normal physiology and pathophysiology. PGE2 acts via four EP receptors (EP1-EP4), which are present on multiple cell types reflecting the diverse functions of PGE2 (Hata and Breyer, 2004). The functions of PGE2 are discussed very controversially as it can have pro- and anti-inflammatory properties. It is an important mediator of active inflammation with local vasodilatory function along with activation and recruitment of macrophages and mast cells (Nakayama et al., 2006; Weller et al., 2007; Yu and Chadee, 1998). But it also has the ability to suppress multiple

Abbreviations: PGE2, prostaglandin E2; AA, arachidonic acid.

is This work was funded by the Konstanz Research School Chemical Biology (KoRSCB) and DFG grant GR 1517/8-1.

* Corresponding author at: University of Konstanz, Department of Biology/Immunology, Universitaetsstr. 10 D-78457 Konstanz, Germany.

Tel.: +49 7531882130 ; fax: +49 7531883102.

E-mail address: Marcus.Groettrup@uni-konstanz.de (M. Groettrup). pro-inflammatory cytokines by inducing anti-inflammatory IL-10 and thus promoting immune suppression (Phipps et al., 1991; Wang et al., 2007). PGE2 modulates the activities of professional APCs such as dendritic cells and macrophages by regulating the production of cytokines by these cells. It plays an important role in the migration of mature DCs by enhancing the signal transduction of the chemokine receptor CCR7 (Scandella et al., 2002). Moreover, PGE2 induces the production of IL-23 and inhibits the release of IL-12 from bone marrow derived dendritic cells (Sheibanie et al., 2004). PGE2 also modulates the proliferation of CD4+ T-cells and their ability to produce cytokines (Choudhry et al., 1999a,b).

$\mathrm{CD} 4^{+} \mathrm{T}$-cells undergo distinct differentiation pathways depending on the kind of cytokine present in the microenvironment. Initially, two effector T-helper subsets were distinguished: Th1 and Th2 cells (Mosmann and Coffman, 1989). Th1 differentiation is promoted by IL-12 with IFN- $\gamma$ as an important effector cytokine while Th2 differentiation is promoted by IL- 4 and IL-10 (Fort et al., 2001; Mosmann and Coffman, 1989). Recently, a distinct $\mathrm{T}$ helper cell subset designated Th17 was characterized in mice (Aggarwal et al., 2003; Cua et al., 2003; Harrington et al., 2005; Langrish et al., 2005; Park et al., 2005) and humans (AcostaRodriguez et al., 2007; Annunziato et al., 2007; Chen and O'Shea, 2008; van Beelen et al., 2007). Th17 cells produce high amounts of IL-17A and IL-17F and are driven by the master transcription factor RORyt (Ivanov et al., 2009). Th17 cells play a role in 
protection against extracellular bacteria and fungi, and are important mediators of autoimmune diseases like rheumatoid arthritis, experimental autoimmune encephalomyelitis, and inflammatory bowel disease (Langrish et al., 2005; Murphy et al., 2003; Yen et al., 2006). IL-23 is a pro-inflammatory cytokine that consists of a unique $\mathrm{p} 19$ subunit and a $\mathrm{p} 40$ subunit, which it shares with IL-12 (Oppmann et al., 2000). IL-23 in combination with IL-1 is sufficient to induce naive human T-cells to produce IL-17A, IL-17F, IL-22 and the transcription factor ROR $\gamma \mathrm{t}$ (Wilson et al., 2007).

PGE2 inhibits the production of IL-12 from monocytes (van der Pouw Kraan et al., 1995) and DCs (Kalinski et al., 1997), thus inhibiting Th1 differentiation and promoting a Th2 response, which is evident by the overproduction of PGE2 in multiple Th2associated diseases, most prominently in atopic dermatitis and asthma (Kapsenberg et al., 1999). PGE2 has also been shown to promote IL-17 producing Th17 through EP2-EP4 signaling by suppressing IL-12 production while enhancing IL-23 production from DC (Boniface et al., 2009; Esaki et al., 2010; Khayrullina et al., 2008; Sheibanie et al., 2007; Yao et al., 2009). Nevertheless, the role of PGE2 as a paracrine mediator of pro-inflammatory and antiinflammatory immune responses has remained a matter of very controversial debate (Kalinski, 2012; Rieser et al., 1997).

Monocytes have a possible role in the acute exacerbation of autoimmune diseases like MS and rheumatoid arthritis (Highton et al., 1989; Imamura et al., 1993). Monocytes activated in a proinflammatory environment can initiate autoimmune diseases by entering the target organs before T-cells, by traversing normal endothelium without help from any other cells (de Jong et al., 1996; Lidington et al., 1998; Stoy, 2002). While there is a positive effect of PGE2 on IL-23 production by DC, the effect of PGE2 on human monocytes in this respect has not been studied in detail. Here we show that PGE2 blocks the production of IL-23 from LPS-stimulated human monocytes and this effect was due to the down-regulation of the p40 subunit of IL-23 and IL-12 at mRNA and protein level. We conclude that PGE2 has the opposite effects on monocytes and DC with respect to IL-23 production emphasizing the cell type dependence of its function.

\section{Material and methods}

\subsection{PBMCs and monocyte isolation}

Human PBMCs were separated from whole blood by standard density gradient centrifugation on Ficoll-Paque ${ }^{\mathrm{TM}}$ Plus (GE Healthcare). For isolation of $\mathrm{CD} 14^{+}$monocytes, PBMCs were incubated with anti-CD14 conjugated microbeads (Miltenyi Biotech) and positively selected for monocytes by magnetic separation using MACS columns (Miltenyi Biotech). Blood donations were conducted after informed consent and were approved by the Institutional Review Board of Konstanz University.

\subsection{Generation of human monocyte derived DCs (MoDCs) and monocyte-derived macrophages}

For generation of MoDCs, isolated monocytes were cultured at $2 \times 10^{6}$ cells $/ \mathrm{ml}$ in AIM-V medium (Invitrogen) along with $50 \mathrm{ng} / \mathrm{ml}$ GM-CSF (Peprotech) and $50 \mathrm{ng} / \mathrm{ml} \mathrm{IL-4} \mathrm{(Peprotech)} \mathrm{for} 5$ days. For generation of monocyte-derived macrophages, isolated monocytes at $2 \times 10^{6}$ cells $/ \mathrm{ml}$ were cultured in AIM-V medium along with $50 \mathrm{ng} / \mathrm{ml} \mathrm{GM}-\mathrm{CSF}$ (Peprotech) for 5 days. The same volume of media along with the same amount of cytokines were added on day 2 to the culture. On day 6 , cells were harvested and stimulated with $5 \mu \mathrm{g} / \mathrm{ml}$ LPS (Sigma) in the presence or absence of $1 \mu \mathrm{g} / \mathrm{ml}$ PGE2 (Sigma). Finally, the supernatant was harvested after $48 \mathrm{~h}$ for analysis by ELISA.

\subsection{Cytokine assays}

Isolated monocytes were cultured in RPMI 1640 (Invitrogen) at $2 \times 10^{6}$ cells $/ \mathrm{ml}$ in the presence or absence of $5 \mu \mathrm{g} / \mathrm{ml}$ LPS (Sigma) under different conditions as indicated in the figures. Supernatants were collected after different time points as indicated and analyzed by ELISA by means of commercially available kits to detect IL-23 (eBioscience), IL-12p70 (eBioscience), IL-6 (BD Pharmingen), IL-10 (BD Pharmingen), and IL-1 $\beta$ (BD Pharmingen) according to the manufacturers' protocols.

\subsection{Cell staining and flow cytometry}

Isolated monocytes were stimulated with $5 \mu \mathrm{g} / \mathrm{ml}$ LPS (Sigma) in the presence or absence of $1 \mu \mathrm{g} / \mathrm{ml}$ PGE2 (Sigma) along with brefeldin A (Sigma) for $18 \mathrm{~h}$. Subsequently, cells were harvested and surface stained with FITC-conjugated CD14 antibody (Miltenyi Biotech). Cells were fixed with Fixation-Permeabilisation buffer (eBioscience) according to the manufacturer's instruction. Cells were then stained intracellularly with efluor 660-conjugated IL23p19 (eBioscience), alexafluor 647-conjugated IL-12p35 (clone SNKY35, eBioscience), and PE-conjugated IL-12/23p40 (clone eBioHP40, eBioscience) in permeabilization buffer overnight at $4{ }^{\circ} \mathrm{C}$. Matured monocyte-derived DCs were surface stained for maturation markers PE-conjugated CD80, PE-conjugated CD83, PE-conjugated CD86, and APC-conjugated CCR7 (all from BD Biosciences). Flow cytometric analyses were performed on a BD FACS Aria $^{\mathrm{TM}}$ IIIu (BD Biosciences) and data were analyzed with Flowjo software (Tree Star).

\subsection{RNA isolation and cDNA synthesis}

Isolated monocytes were stimulated with $5 \mu \mathrm{g} / \mathrm{ml}$ LPS (Sigma) in the presence or absence of $1 \mu \mathrm{g} / \mathrm{ml}$ PGE2 for $5 \mathrm{~h}$. Cells were harvested thereafter and processed for total RNA isolation by using the Nucleospin ${ }^{\circledR}$ RNA isolation kit (Macherey Nagel) as per the manufacturer's protocol. $1 \mu \mathrm{g}$ of RNA was used for synthesizing cDNA using the reverse transcription kit from Promega. The resulting cDNA was used for real time RT-PCR.

\subsection{Real time RT-PCR}

Real time RT-PCR was performed with the Light Cycler (Roche) and the Light Cycler-DNA Master SYBR Green I kit (Roche) according to the manufacturer's protocol. After initial denaturation for $30 \mathrm{~s}$ at $95^{\circ} \mathrm{C}$, thermal cycling was performed for 38 cycles with steps of $95^{\circ} \mathrm{C}$ for $10 \mathrm{~s}, 60^{\circ} \mathrm{C}$ for $7 \mathrm{~s}$, and $72{ }^{\circ} \mathrm{C}$ for $8 \mathrm{~s}$, with fluorescence being read at the end of each cycle. The following oligonucleotides were used as primers for the PCR: IL-23p19 fwd-5'GCTTCAAAATCCTTCGCAG 3'; IL-23p19 rev-5'TATCTGAGTGCCATCCTTGAG 3'; IL-12/23p40 fwd-5'GTCAGAGGGGACAACAAGGA $\quad 3^{\prime}$; $\quad$ IL-12/23p40 rev5'TGATGAAGAAGCTGCTGGTG 3'. The data analysis was performed with Light Cycler software 3. The values were normalized to 18S rRNA which was used as a reference. All PCR products were analyzed by determination of melting profiles as well as by agarose gel electrophoresis.

\subsection{Statistical analysis}

The statistical significance of the differences was determined using Student's t-test. The Graph Pad Prism software (version 4.03) (Graph Pad) was used for all statistical analyses. 


\section{Results}

\subsection{PGE2 inhibits IL-23 production by human PBMCs and isolated monocytes}

To assess the effect of the lipid immunomodulator PGE2 on the ability of human monocytes to produce IL-23, human PBMCs were isolated from whole blood and stimulated with LPS in the presence or absence of PGE2 for $24 \mathrm{~h}$. The analysis of the supernatant by ELISA showed that PGE2 strongly inhibited IL-23 production from PBMCs whereas the solvent control did not show any effect (Fig. 1A). In contrast, there was no effect of PGE2 on IL-6 production (Fig. 1A), documenting the specificity of PGE2 in down-regulating IL-23 production. To investigate whether the ability of PGE2 to block IL-23 production was a direct effect on monocytes, CD14 ${ }^{+}$monocytes were magnetically isolated from PBMCs to a purity of more than $95 \%$ and stimulated in vitro with LPS for $24 \mathrm{~h}$ in the presence or absence of PGE2. As evidenced for PBMCs before, PGE2 largely inhibited IL-23 production from isolated monocytes as well (Fig. 1B) thus indicating a direct effect of PGE2 on monocytes in blocking IL-23 production. Again, IL-6 production from monocytes was not affected by PGE2 (Fig. 1B), illustrating the specific effect of PGE2 on the suppression of IL-23 production. We also tested the effect of PGE2 on IL-23 production by isolated monocytes after stimulation with poly I:C and found that also with this TLR ligand PGE2 led to a down regulation of IL-23 production while the IL-6 production remained unaltered (Fig. 1C).

\subsection{PGE2 promotes IL-23 production by monocyte derived DCS but not by monocyte derived macrophages}

Since PGE2 has been reported to up-regulate the production of IL-23 by monocyte derived DCs (MoDCs)(Sheibanie et al., 2004, 2007), we decided to compare MoDCs and monocyte derived macrophages in this respect. To this aim we differentiated monocytes to immature MoDCs with GMCSF and IL- 4 for 5 days followed by stimulation with LPS in the presence or absence of PGE2 for $48 \mathrm{~h}$ to induce maturation. Subsequently, the supernatants were collected and analyzed for IL-23 and IL- 6 production by ELISA and the cells were characterized for the surface expression of DC maturation markers. PGE2 led to an up-regulation of surface markers CD80, CD83, CD86, and CCR7 on matured MoDCs (Supplementary Fig. S1). In accordance with previous studies (Sheibanie et al., 2004, 2007), PGE2 up-regulated the production of IL-23 and IL-6 from immature MoDCs upon LPS stimulation (Fig. 2A). We also cultured monocytes with GMCSF only to differentiate them to monocytederived macrophages and then stimulated them with LPS in the presence or absence of PGE2 for $48 \mathrm{~h}$. The supernatants were collected thereafter and analyzed for IL-23 and IL- 6 content by ELISA. PGE2 did not affect the production of IL-23 and IL-6 from monocyte derived macrophages upon LPS stimulation (Fig. 2B). These data shows a cell context specific effect of PGE2 with respect to governing the production of IL-23.

\subsection{PGE2 inhibits IL-23 and IL-12 production from isolated} monocytes through down-regulation of the common p40 subunit both at the MRNA and protein level

IL-23 is a heterodimer of the subunits p19 and p40. Since PGE2 was leading to a down-regulation of IL-23 production by monocytes, we wanted to investigate the effect of PGE2 on the individual p19 and p40 subunits of IL-23. To this aim, we performed intracellular staining for the individual subunits of IL-23, i.e. p19 and $\mathrm{p} 40$. Isolated monocytes in the presence of brefeldin A were stimulated with LPS in the presence or absence of PGE2 for $18 \mathrm{~h}$. They were subsequently surface stained for CD14 followed by intracellular staining for p19 and p40. Sorted CD14 positive cells showed a CD14high and CD14low population (Supplementary Fig. S2). Upon LPS stimulation, there was an up-regulation of CD14 and an increase in the CD14high population. We consistently observed an increase in p19 and p40 producing cells upon LPS stimulation in both the CD14high and CD14low populations (Supplementary Fig. S2). PGE2 led to a significant down-regulation of $\mathrm{p} 40$ producing cells in both the CD14high and CD14low population (Supplementary Fig. S2). We also performed a co-staining for p19 and p40, and found that PGE2 led to a significant down-regulation of p19/p40 double positive cells, which could be functional IL-23 producing cells (Fig. 3A, top). Since the p40 subunit is also shared by IL-12, we wanted to investigate whether the suppression of $\mathrm{p} 40$ by PGE2 is also affecting IL-12 production by monocytes. Hence, co-stainings for the individual subunits of IL-12 p35 and p40 were performed (Fig. 3A, bottom). PGE2 also led to a significant down-regulation of p35/p40 double positive cells, which could be functional IL-12 producing cells (Fig. 3A). To determine whether the suppression of p40 also occurred on mRNA level, real time RT-PCR analyses were performed. PGE2 led to a significant down-regulation of the mRNA for the p40 subunit while there was no significant effect on the level of mRNA for the p19 subunit (Fig. 3B).

\subsection{PGE2 acts in a concentration dependent manner to inhibit IL-12 and IL-23 production by isolated monocytes after different time points of LPS stimulation}

In order to confirm the effect of PGE2 on the production of the heterodimeric IL-12 p70 complex by LPS-stimulated monocytes on the protein level analyses of supernatants by ELISA were performed. For this analysis isolated human monocytes were stimulated with LPS and IFN- $\gamma$ in the presence or absence of PGE2. The supernatants were collected after 24 and $48 \mathrm{~h}$ of stimulation and the concentrations of IL-12p70 were determined with a p70 specific antibody detecting the $\mathrm{p} 35 / \mathrm{p} 40$ heterodimer. There was a significant upregulation of IL-12 production by monocytes after LPS and IFN- $\gamma$ stimulation (Fig. 4A, bottom). The IL-12 concentration reached its maximum after $24 \mathrm{~h}$ of stimulation and was significantly downregulated by the effect of PGE2 at both 24 and $48 \mathrm{~h}$ post stimulation without any effect on IL-6 production (Fig. 4A). In addition to IL12, we also measured the content of the IL-23 p19/p40 heterodimer in the supernatants after 24 and $48 \mathrm{~h}$ post LPS stimulation in the presence or absence of PGE2. IL-23 production peaked at $24 \mathrm{~h}$ and its concentration started to decline in the supernatant thereafter, which could be due to its consumption by cells in the culture (Fig. 4A, top). PGE2 resulted in a down-regulation of IL-23 at 24 and $48 \mathrm{~h}$ post stimulation without any effect on IL-6 production. We also investigated on the effect of PGE2 on IL-12 and IL-23 production by isolated monocytes at different concentrations of PGE2. PGE2 inhibited IL-12 and IL-23 production by isolated monocytes in a dose-dependent manner upon LPS stimulation (Fig. 4B). Even at the lowest concentration of $10 \mathrm{ng} / \mathrm{ml} \mathrm{PGE2}$, there was a nearly $50 \%$ down-regulation of IL-12 and IL-23 while there was no effect on IL-6 levels in the supernatants at all concentrations of PGE2 tested (Fig. 4B).

\subsection{PGE2 inhibits IL-12 and IL-23 production from isolated monocytes in a cAMP dependent manner}

Monocytes exclusively express the PGE receptors EP2 and EP4, the stimulation of which leads to the enhancement of intracellular cAMP levels (Panzer and Uguccioni, 2004). We investigated whether the suppressive effect of PGE2 on IL-12 and IL-23 production by monocytes was cAMP mediated by applying the cAMP enhancer forskolin. We quantified IL-12 and IL-23 production from isolated monocytes stimulated with LPS in the presence or absence 

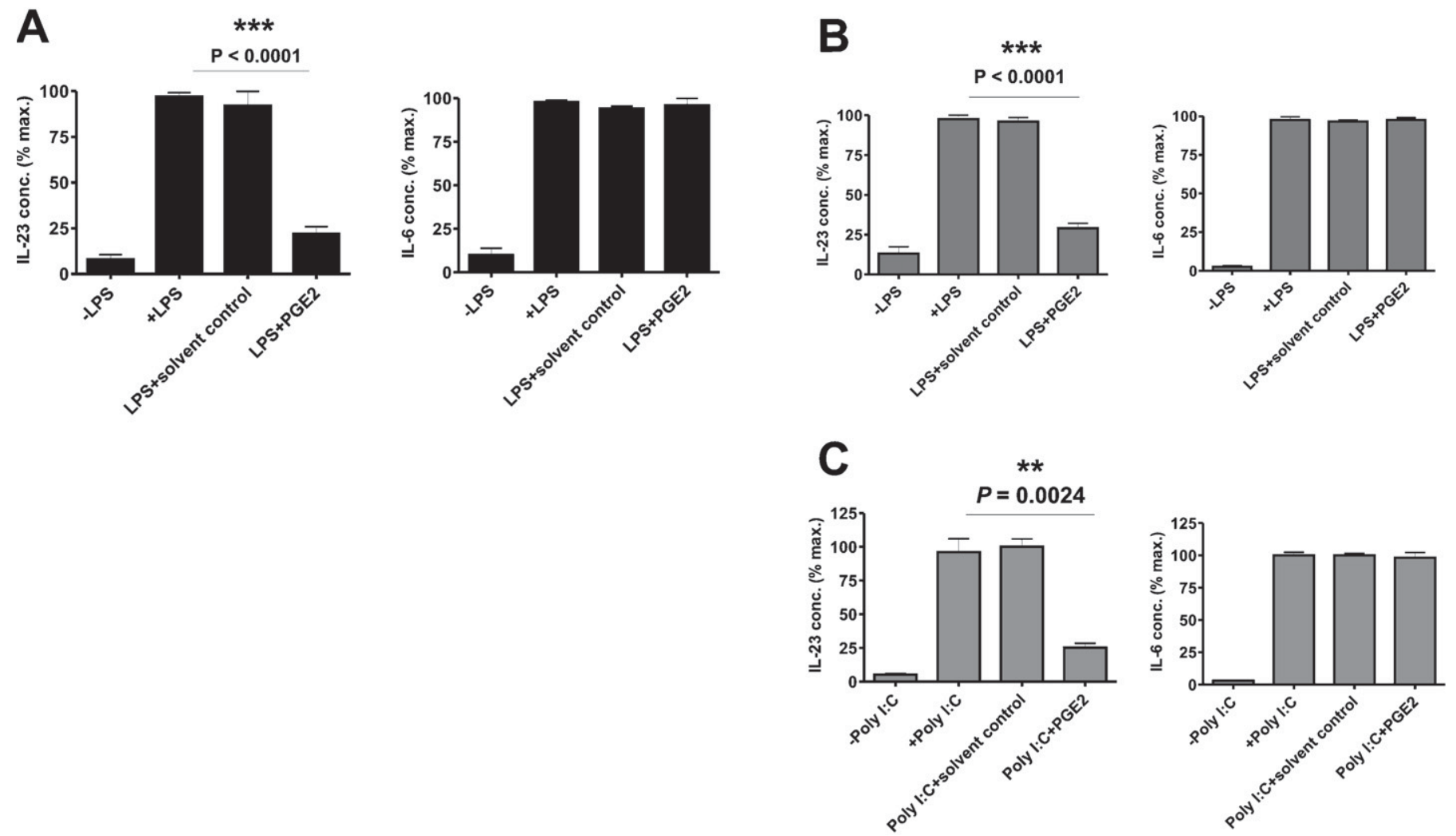

Fig. 1. Effect of PGE2 on IL-23 and IL-6 production by human PBMCs and isolated monocytes. (A) Human PBMCs and (B) isolated monocytes ( $2 \times 10^{6}$ cell/ml) were stimulated with $5 \mu \mathrm{g} / \mathrm{ml}$ LPS in the presence or absence of $1 \mu \mathrm{g} / \mathrm{ml}$ of PGE2 and supernatants were collected after $24 \mathrm{~h}$. IL-23 and IL-6 concentrations were measured in the supernatant by ELISA. Error bars represent standard deviations from 3 different donors for PBMCs and 5 different donors for isolated monocytes. (C) Isolated monocytes were stimulated with polyI:C in the presence and absence of PGE2 and supernatants were collected after $24 \mathrm{~h}$. IL-23 and IL-6 concentrations were measured in the supernatant by ELISA. Error bars represent standard deviations from 3 different donors.

A
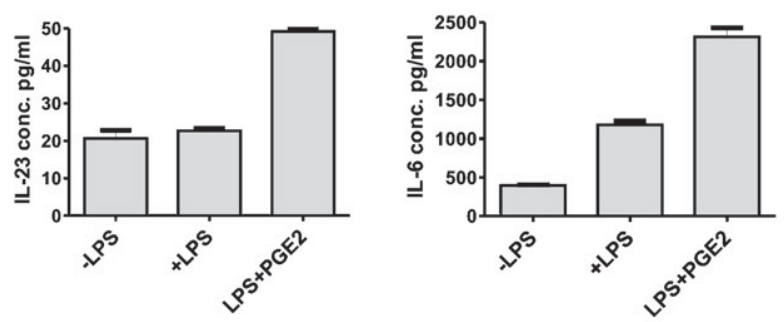

Donor 2

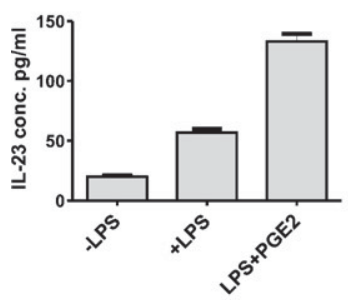

Donor 1

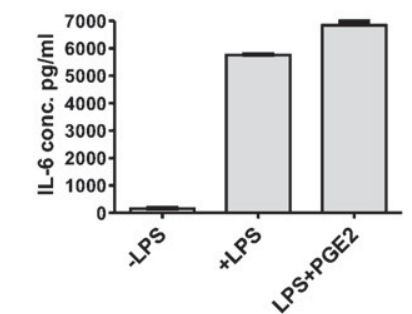

B
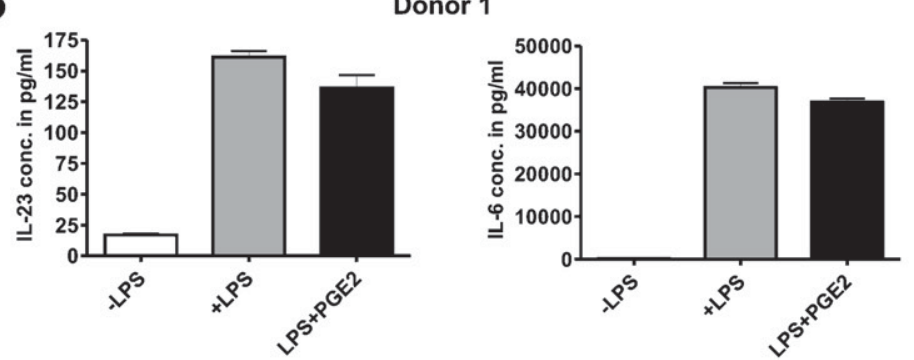

Donor 2

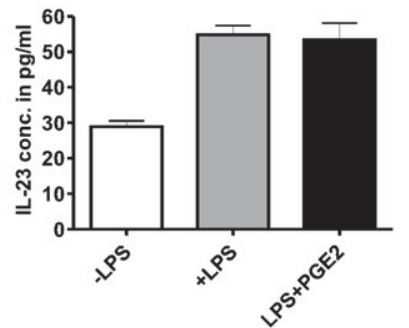

Fig. 2. Influence of PGE2 on IL-23 and IL-6 production from human monocyte derived DCs and monocyte derived macrophages. (A) Immature monocyte derived DCs and (B) monocyte derived macrophages were stimulated with LPS in the presence or absence of $1 \mu \mathrm{g} / \mathrm{ml}$ PGE2 and supernatants were collected after 48 h. IL-23 and IL-6 concentrations were measured by ELISA. Error bars represent standard deviations of triplicate readings from the same donor. Data from two different donors are shown. 
A -LPS
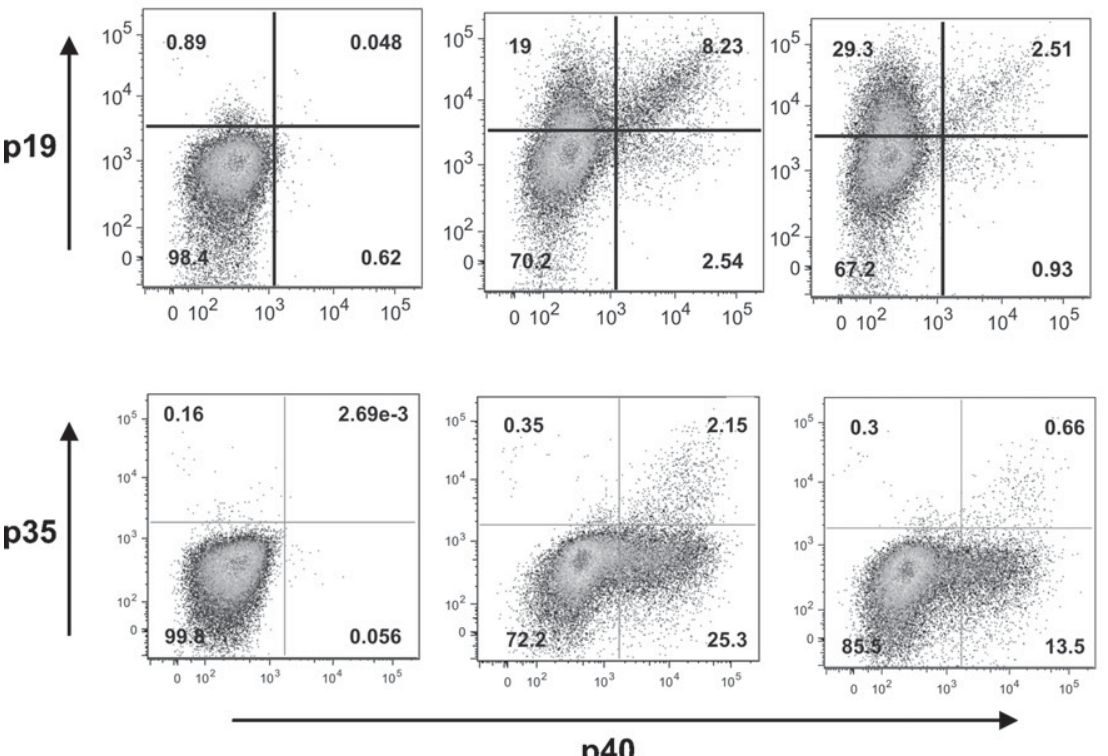

B
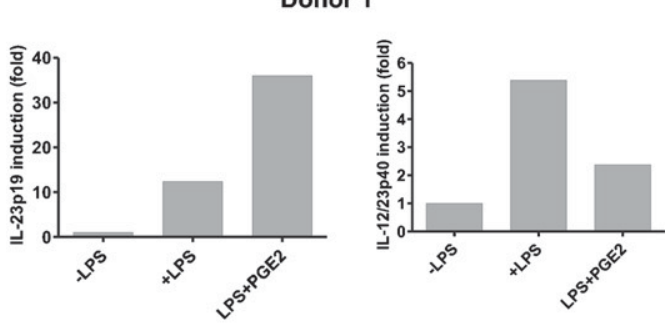

Donor 2
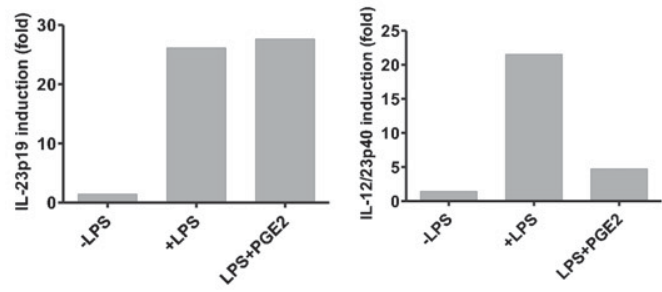

Donor 3
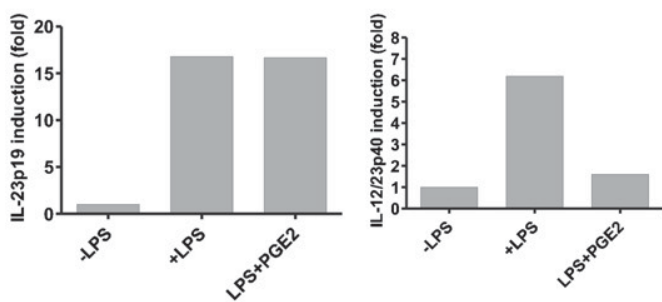

Fig. 3. Effect of PGE 2 on IL- 23 and IL-12 production from isolated monocytes at the mRNA and protein level. Isolated human monocytes ( $2 \times 10^{6}$ cell/ml) treated with brefeldin A were stimulated with LPS in the presence or absence of $1 \mu \mathrm{g} / \mathrm{ml}$ PGE2. (A) Cells were harvested after $15 \mathrm{~h}$ and surface stained for CD14 followed by intracellular co-staining for the IL-12 and IL-23 subunits p35, p40 and p19, respectively, and then analyzed by flow cytometry. Recombinant human IFN- $\gamma$ was added to the culture along with LPS for induction of IL-12. The shown dot plots are representative for three independent experiments from different donors with similar outcomes. (B) Isolated monocytes $\left(2 \times 10^{6} \mathrm{cell} / \mathrm{ml}\right)$ were stimulated with LPS in the presence or absence of PGE2 for $4 \mathrm{~h}$ and subsequently cells were harvested followed by RNA isolation and real time RT-PCR for IL-23p19 and the common p40 subunit of IL-12 and IL-23. Data from three different donors are shown.

of PGE2 and also in the presence or absence of $50 \mu \mathrm{M}$ forskolin. PGE2 was leading to a down-regulation of IL-12 and IL-23 as observed in previous experiments (Fig. 5A and B). Forskolin was able to mimic the effect of PGE2 as the supplementation of the culture medium with $50 \mu \mathrm{M}$ forskolin led to a down-regulation of IL-12 and IL-23 in the supernatant of LPS stimulated monocytes which was similar to that of PGE2 (Fig. 5A and B). Again, there was no effect on IL-6 content after forskolin treatment (Fig. 5A and B). Our data strongly suggests that PGE2 acting through EP2 and EP4 receptors leads to an elevation of cAMP level and this in turn appears to be responsible for the down-regulation of IL-12 and IL-23 production by isolated monocytes.

\section{Discussion}

IL-23 is a heterodimeric cytokine belonging to the IL-12 family of cytokines. IL-23 is constituted from a unique p19 subunit and the $\mathrm{p} 40$ subunit, which it shares with the heterodimeric cytokine IL-12. IL-23 plays a crucial role in the differentiation and maintenance of human Th17 cells and therefore has been implicated in several inflammatory autoimmune disorders. The effect of the non-cytokine immunomodulator prostaglandin E2 on IL-23 production by DCs has been studied extensively but its effect on human monocytes, which also play an important role in the progression of autoimmune disorders by secreting pro-inflammatory cytokines (Chofflon et al., 1992; Imamura et al., 1993), has not been investigated in detail.
We have shown in this study that PGE2 is affecting the expression of the common p40 subunit in activated monocytes leading to its suppression both at mRNA and protein level, which was responsible for the down-regulation of IL-12 and IL-23 in monocytes. PGE2 acts through EP2 and EP4 receptors leading to the enhancement of cAMP levels. The effect of PGE2 of blocking IL-12 and IL-23 production from both total PBMCs and isolated monocytes was attributed to the enhancement of cAMP levels since forskolin, which is a pharmacologic enhancer of intracellular cAMP, was able to show the same effect like that of PGE2 in blocking these pro-inflammatory cytokines. LPS signaling in monocytes induces a wide range of signaling pathways and transcription factors, which include MAP kinase and NF- $\mathrm{kB}$ signaling pathways. Both $\mathrm{p} 38 \mathrm{MAP}$ kinase and NF$\kappa \mathrm{B}$ are important in LPS mediated transcriptional induction of the p40 gene; in particular the NF- $\kappa$ B component c-Rel plays an important role in the induction of IL-23 (Zhang et al., 2000). However, a detailed experimental promoter analysis of $\mathrm{p} 40$ is needed to clarify which regulatory proteins and transcription factors possess binding sites in the promoter region of the p 40 gene. It would be interesting to investigate the effect of PGE2 on those regulatory proteins, which might be responsible for the observed down-regulation of p40 mRNA levels. It would also be interesting to look at the effect of PGE2 on other MAP kinase pathways activated by LPS stimulation like the JNK and ERK pathways. It has been shown earlier that cAMP inhibits p38 MAP kinase activity leading to the downregulation of IL-12p40 (Feng et al., 2002; Zhang et al., 2006), which could be a possible mechanism underlying our finding of the fulminant down-regulation of the p40 mRNA. It would be interesting 

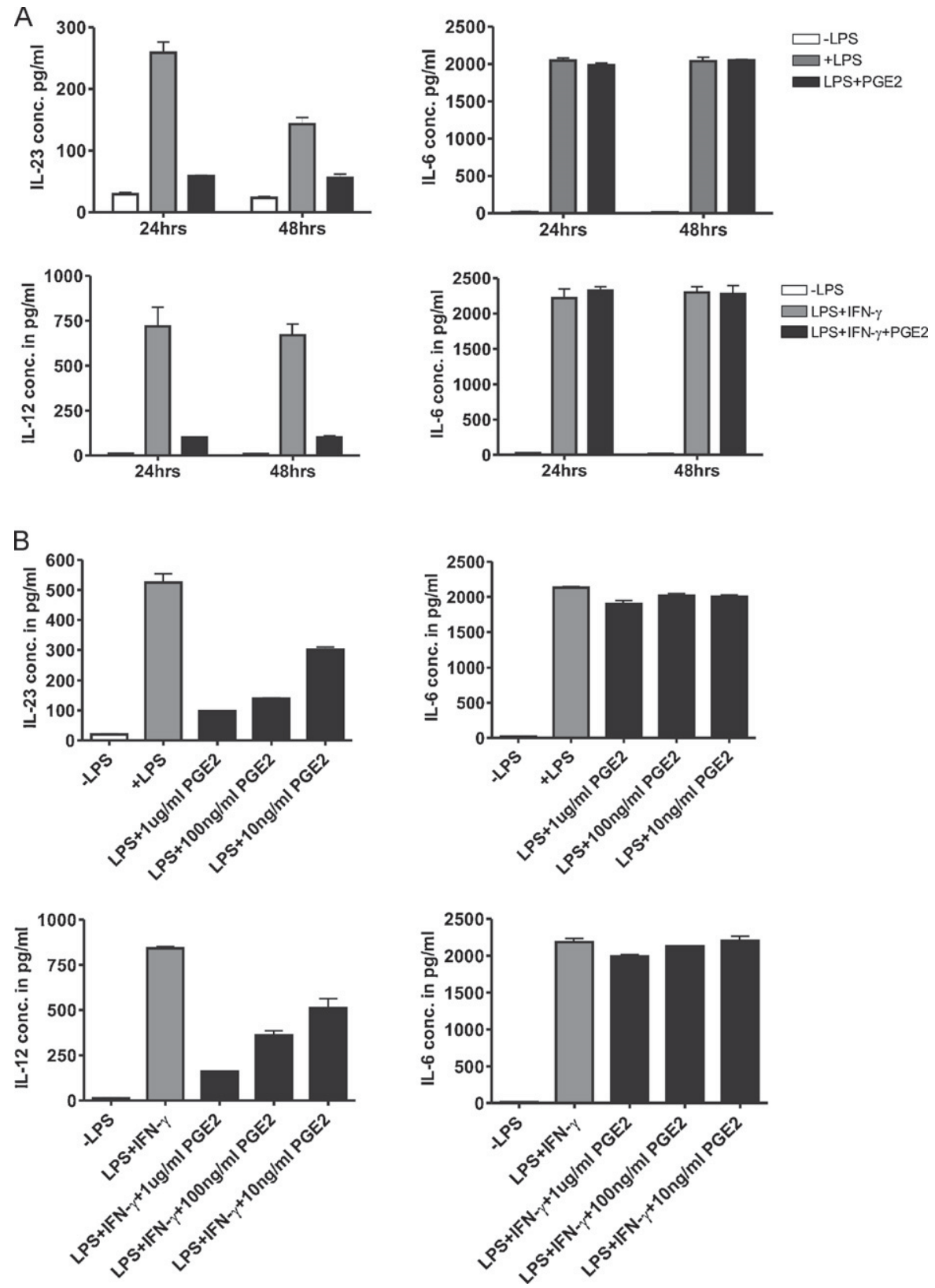

Fig. 4. Dose dependent effect of PGE2 on IL-12 and IL-23 production by isolated human monocytes after different time points of LPS stimulation. (A) Isolated monocytes $\left(2 \times 10^{6} \mathrm{cell} / \mathrm{ml}\right)$ were stimulated with LPS in the presence or absence of $1 \mu \mathrm{g} / \mathrm{ml}$ PGE2. Supernatants were collected after 24 and $48 \mathrm{~h}$. ELISAs were performed for IL-23 (p19/p40 dimer) and IL-12 (p35/p40 dimer). Error bars represent standard deviation of triplicate readings. The data is representative of three independent experiments with different donors. (B) Isolated monocytes $\left(2 \times 10^{6} \mathrm{cell} / \mathrm{ml}\right)$ were stimulated with LPS in the presence or absence of different concentrations of PGE2 for $24 \mathrm{~h}$. Supernatants were analyzed for the concentration of IL-23 and IL-12 by ELISA. Error bars represent standard deviations of triplicate readings from the same donor. Data is representative of at least three independent experiments with different donors.

to investigate further downstream of cAMP, the cell context specific effect of PGE2 on monocytes and monocytes derived DCs with respect to IL-23 production. cAMP signals through PKA and Epac, the guanine nucleotide exchange factor for the small GTPase Rap1 (de Rooij et al., 1998) and the crosstalk between cAMP versus PKA and Epac signaling is responsible for DC maturation (Garay et al., 2010). It could be possible that cAMP signaling via PGE2 and its cross talk with PKA and Epac signaling is operating differentially in the two cell types, i.e. monocytes and monocytes derived DCs.

IL-10 is a potent inhibitor of many of the functions of monocytes, including oxidative burst, nitric oxide production, phagocytosis, and the production of pro-inflammatory cytokines like IL-12
(Bogdan et al., 1991; Fiorentino et al., 1991). It has been shown that IL-10 inhibits the transcription of inflammatory cytokines produced by monocytes upon LPS stimulation (Wang et al., 1994), and IL-1 $\beta$ is involved in the production of IL- 12 by human monocytes (van de Wetering et al., 2009). Therefore, we have investigated in this study whether the effect of PGE2 of blocking IL-12 and IL-23 production was mediated by altering the expression of IL-10 and IL$1 \beta$ in monocytes, but we could not detect any effect of PGE2 on IL-10 and IL-1 $\beta$ production by isolated monocytes upon LPS stimulation (Supplementary Fig. S3). Moreover, as concluded from our intracellular staining experiments and real time RT-PCR analyses of isolated monocytes, PGE2 does not seem to act via the induction of any other 
A
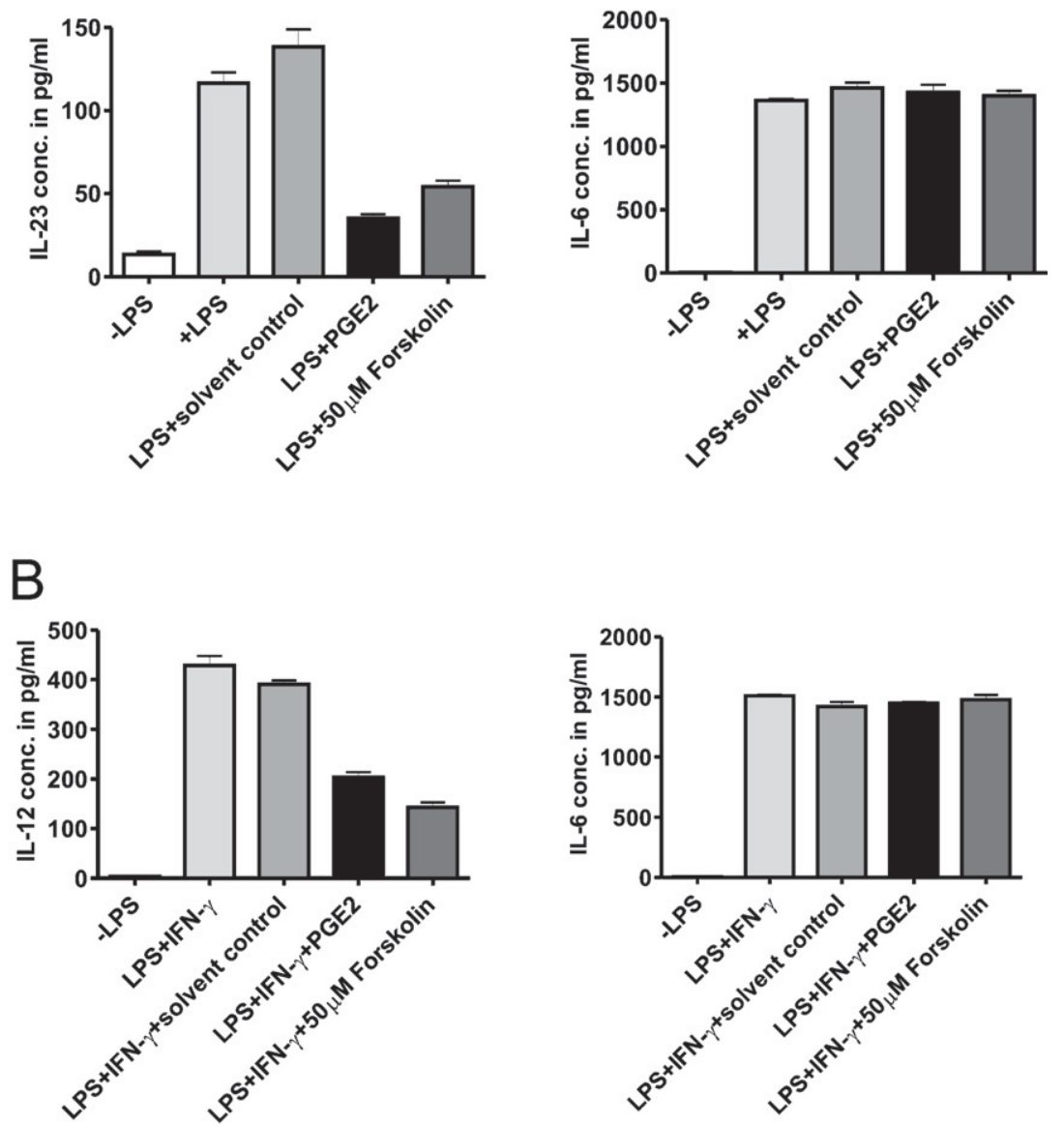

Fig. 5. The effect of PGE2 on IL-23 and IL-12 down-regulation in isolated monocytes is cAMP mediated. Isolated human monocytes $\left(2 \times 10^{6}\right.$ cell/ml) were stimulated with LPS and IFN- $\gamma$ under different conditions in the presence of $1 \mu \mathrm{g} / \mathrm{ml}$ of PGE2 or $50 \mu \mathrm{M}$ forskolin as indicated for 24 h. Supernatants were collected thereafter and ELISAs were performed for quantitating (A) IL-23 and (B) IL-12. Data is representative of at least three independent experiments from different donors.

soluble mediator or cytokine, which might be responsible for the reduced IL-12 and IL-23 production by monocytes. Instead, our data strongly suggest that PGE2 exerts this effect directly by binding to EP2 and/or EP4 receptors on highly purified monocytes leading to the enhancement of intracellular cAMP levels which in turn most likely suppress the transcription of these pro-inflammatory cytokines produced by monocytes upon LPS stimulation. We should point out, however, that we have not ruled out that the reduction in the steady state level of p40 mRNA is due to an accelerated degradation of the mRNA encoding $\mathrm{p} 40$ rather than a reduced transcription of the $\mathrm{p} 40$ gene.

PGE2 has been known for its unique status of a proinflammatory factor with both immunosuppressive as well as immuno-stimulating activity. PGE2 promotes Th17 differentiation by inducing IL-23 production by DCs. It would be interesting to look at the effect of PGE2 on Th1 and Th17 differentiation in the context of monocytes.

\section{Conclusion}

Our data strongly suggest that PGE2 affects T-helper cell differentiation in a cell context dependent manner. When PGE2 is acting on DCs it promotes Th17 differentiation whereas when it is acting on monocytes it inhibits both Th1 and Th17 differentiation due to its inhibitory effect on IL-12 and IL-23 production by human monocytes, respectively. This dichotomy between monocytes in the peripheral blood and mature DCs in secondary lymphoid organs may be a means to counteract an overshooting pro-inflammatory response and cytokine storm in the circulation whereas DCs licensed by TLR ligands and PGE2 are allowed to promote a pro-inflammatory response in secondary lymphoid organs. This dichotomy and cell type as well as differentiation state specific consequences of PGE2 stimulation, especially when comparing monocytes with DCs, is an important paradigm, which may help to reconcile controversial findings on the role of PGE2 in the regulation of cytokines of the IL12 family.

\section{Authors' contribution}

K.W.K. designed and performed all experiments, analyzed the data, and wrote the manuscript.; M.G. provided advice for experimental design, supervised the experiments and refined the manuscript.

\section{Conflict of interest}

The authors declare no competing financial interests. 


\section{Acknowledgements}

We thank Michael Basler for useful discussion and suggestions. We also thank Margit Richter for experimental advice and help. We acknowledge Marc Mueller, Ilona Kindinger, and Markus Bruckner for their help with blood donations. Ulrike Beck is acknowledged for excellent technical assistance. KWK holds a fellowship of KoRS-CB and Zukunftskolleg at the University of Konstanz.

\section{Appendix A. Supplementary data}

Supplementary data associated with this article can be found, in the online version, at http://dx.doi.org/10.1016/ j.molimm.2012.08.014.

\section{References}

Acosta-Rodriguez, E.V., Napolitani, G., Lanzavecchia, A., Sallusto, F., 2007. Interleukins 1 beta and 6 but not transforming growth factor-beta are essential for the differentiation of interleukin 17-producing human T helper cells. Nature Immunology 8, 942-949.

Aggarwal, S., Ghilardi, N., Xie, M.H., de Sauvage, F.J., Gurney, A.L., 2003. Interleukin23 promotes a distinct CD4T cell activation state characterized by the production of interleukin-17. Journal of Biological Chemistry 278, 1910-1914.

Annunziato, F., Cosmi, L., Santarlasci, V., Maggi, L., Liotta, F., Mazzinghi, B., Parente, E., Fili, L., Ferri, S., Frosali, F., Giudici, F., Romagnani, P., Parronchi, P., Tonelli, F., Maggi, E., Romagnani, S., 2007. Phenotypic and functional features of human Th17 cells. Journal of Experimental Medicine 204, 1849-1861.

Bogdan, C., Vodovotz, Y., Nathan, C., 1991. Macrophage deactivation by interleukin 10. Journal of Experimental Medicine 174, 1549-1555.

Boniface, K., Bak-Jensen, K.S., Li, Y., Blumenschein, W.M., McGeachy, M.J., McClanahan, T.K., McKenzie, B.S., Kastelein, R.A., Cua, D.J., de Waal Malefyt, R., 2009. Prostaglandin E2 regulates Th17 cell differentiation and function through cyclic AMP and EP2/EP4 receptor signaling. Journal of Experimental Medicine 206, 535-548.

Chen, Z., O'Shea, J.J., 2008. Regulation of IL-17 production in human lymphocytes. Cytokine 41, 71-78.

Chofflon, M., Juillard, C., Juillard, P., Gauthier, G., Grau, G.E., 1992. Tumor necrosis factor alpha production as a possible predictor of relapse in patients with multiple sclerosis. European Cytokine Network 3, 523-531.

Choudhry, M.A., Ahmed, Z., Sayeed, M.M., 1999a. PGE(2)-mediated inhibition of T cell p59(fyn) is independent of cAMP. American Journal of Physiology 277, C302-C309.

Choudhry, M.A., Hockberger, P.E., Sayeed, M.M., 1999b. PGE2 suppresses mitogeninduced Ca2+ mobilization in T cells. American Journal of Physiology 277, R1741-R1748.

Cua, D.J., Sherlock, J., Chen, Y., Murphy, C.A., Joyce, B., Seymour, B., Lucian, L., To, W., Kwan, S., Churakova, T., Zurawski, S., Wiekowski, M., Lira, S.A., Gorman, D. Kastelein, R.A., Sedgwick, J.D., 2003. Interleukin-23 rather than interleukin-12 is the critical cytokine for autoimmune inflammation of the brain. Nature 421 , 744-748.

de Jong, A.L., Green, D.M., Trial, J.A., Birdsall, H.H., 1996. Focal effects of mononuclear leukocyte transendothelial migration: TNF-alpha production by migrating monocytes promotes subsequent migration of lymphocytes. Journal of Leukocyte Biology 60, 129-136.

de Rooij, J., Zwartkruis, F.J., Verheijen, M.H., Cool, R.H., Nijman, S.M., Wittinghofer, A., Bos, J.L., 1998. Epac is a Rap1 guanine-nucleotide-exchange factor directly activated by cyclic AMP. Nature 396, 474-477.

Esaki, Y., Li, Y., Sakata, D., Yao, C., Segi-Nishida, E., Matsuoka, T., Fukuda, K., Narumiya, S., 2010. Dual roles of PGE2-EP4 signaling in mouse experimental autoimmune encephalomyelitis. Proceedings of the National Academy of Sciences of the United States of America 107, 12233-12238.

Feng, W.G., Wang, Y.B., Zhang, J.S., Wang, X.Y., Li, C.L., Chang, Z.L., 2002. cAMP elevators inhibit LPS-induced IL-12 p40 expression by interfering with phosphorylation of p38 MAPK in murine peritoneal macrophages. Cell Research 12 331-337.

Fiorentino, D.F., Zlotnik, A., Vieira, P., Mosmann, T.R., Howard, M., Moore, K.W., O'Garra, A., 1991. IL-10 acts on the antigen-presenting cell to inhibit cytokine production by Th1 cells. Journal of Immunology 146, 3444-3451.

Fort, M.M., Cheung, J., Yen, D., Li, J., Zurawski, S.M., Lo, S., Menon, S., Clifford, T., Hunte, B., Lesley, R., Muchamuel, T., Hurst, S.D., Zurawski, G., Leach, M.W., Gorman, D.M., Rennick, D.M., 2001. IL-25 induces IL-4, IL-5, and IL-13 and Th2-associated pathologies in vivo. Immunity 15, 985-995.

Garay, J., D’Angelo, J.A., Park, Y., Summa, C.M., Aiken, M.L., Morales, E., Badizadegan, K., Fiebiger, E., Dickinson, B.L., 2010. Crosstalk between PKA and Epac regulates the phenotypic maturation and function of human dendritic cells. Journal of Immunology 185, 3227-3238.

Harrington, L.E., Hatton, R.D., Mangan, P.R. Turner, H., Murphy, T.L., Murphy, K.M. Weaver, C.T., 2005. Interleukin 17-producing CD4 ${ }^{+}$effector T cells develop via a lineage distinct from the T helper type 1 and 2 lineages. Nature Immunology 6 , 1123-1132.
Hata, A.N., Breyer, R.M., 2004. Pharmacology and signaling of prostaglandin receptors: multiple roles in inflammation and immune modulation. Pharmacology \& Therapeutics 103, 147-166.

Highton, J. Smith, M., Bradley, J. 1989. Cells of the monocyte-macrophage series in peripheral blood and synovial fluid in inflammatory arthritis. A preliminary study of cellular phenotype. Scandinavian Journal of Rheumatology 18, 393-398.

Imamura, K., Suzumura, A., Hayashi, F., Marunouchi, T., 1993. Cytokine production by peripheral blood monocytes/macrophages in multiple sclerosis patients. Acta Neurologica Scandinavica 87, 281-285.

Ivanov, I.I., Atarashi, K., Manel, N., Brodie, E.L., Shima, T., Karaoz, U., Wei, D., Goldfarb, K.C., Santee, C.A., Lynch, S.V., Tanoue, T., Imaoka, A., Itoh, K., Takeda, K., Umesaki, Y., Honda, K., Littman, D.R., 2009. Induction of intestinal Th17 cells by segmented filamentous bacteria. Cell 139, 485-498.

Kalinski, P., 2012. Regulation of immune responses by prostaglandin E2. Journal of Immunology 188, 21-28.

Kalinski, P., Hilkens, C.M., Snijders, A., Snijdewint, F.G., Kapsenberg, M.L., 1997. IL-12deficient dendritic cells, generated in the presence of prostaglandin E2, promote type 2 cytokine production in maturing human naive T helper cells. Journal of Immunology 159, 28-35.

Kapsenberg, M.L., Hilkens, C.M., Wierenga, E.A., Kalinski, P., 1999. The paradigm of type 1 and type 2 antigen-presenting cells. Implications for atopic allergy. Clinical and Experimental Allergy 29 (Suppl. 2), 33-36.

Khayrullina, T., Yen, J.H., Jing, H., Ganea, D., 2008. In vitro differentiation of dendritic cells in the presence of prostaglandin E2 alters the IL-12/IL-23 balance and promotes differentiation of Th17 cells. Journal of Immunology 181, 721-735.

Langrish, C.L., Chen, Y., Blumenschein, W.M., Mattson, J., Basham, B., Sedgwick, J.D., McClanahan, T., Kastelein, R.A., Cua, D.J., 2005. IL-23 drives a pathogenic T cell population that induces autoimmune inflammation. Journal of Experimental Medicine 201, 233-240.

Lidington, E.A., McCormack, A.M., Yacoub, M.H., Rose, M.L., 1998. The effects of monocytes on the transendothelial migration of T lymphocytes. Immunology 94, 221-227.

Mosmann, T.R., Coffman, R.L., 1989. TH1 and TH2 cells: different patterns of lymphokine secretion lead to different functional properties. Annual Review of Immunology 7, 145-173.

Murphy, C.A., Langrish, C.L., Chen, Y, Blumenschein, W., McClanahan, T., Kastelein, R.A., Sedgwick, J.D., Cua, D.J., 2003. Divergent pro- and antiinflammatory roles for IL-23 and IL-12 in joint autoimmune inflammation. Journal of Experimental Medicine 198, 1951-1957.

Nakayama, T., Mutsuga, N., Yao, L., Tosato, G., 2006. Prostaglandin E2 promotes degranulation-independent release of MCP-1 from mast cells. Journal of Leukocyte Biology 79, 95-104.

Oppmann, B., Lesley, R., Blom, B., Timans, J.C., Xu, Y., Hunte, B., Vega, F., Yu, N., Wang, J., Singh, K., Zonin, F., Vaisberg, E., Churakova, T., Liu, M., Gorman, D., Wagner, J., Zurawski, S., Liu, Y., Abrams, J.S., Moore, K.W., Rennick, D., de Waal-Malefyt, R., Hannum, C., Bazan, J.F., Kastelein, R.A., 2000. Novel p19 protein engages IL-12p40 to form a cytokine, IL-23, with biological activities similar as well as distinct from IL-12. Immunity $13,715-725$.

Panzer, U., Uguccioni, M., 2004. Prostaglandin E2 modulates the functional responsiveness of human monocytes to chemokines. European Journal of Immunology 34, 3682-3689.

Park, H., Li, Z., Yang, X.O., Chang, S.H., Nurieva, R., Wang, Y.H., Wang, Y., Hood, L., Zhu, Z., Tian, Q., Dong, C., 2005. A distinct lineage of CD4T cells regulates tissue inflammation by producing interleukin 17 . Nature Immunology 6, 1133-1141.

Phipps, R.P., Stein, S.H., Roper, R.L., 1991. A new view of prostaglandin E regulation of the immune response. Immunology Today 12, 349-352.

Rieser, C., Bock, G., Klocker, H., Bartsch, G., Thurnher, M., 1997. Prostaglandin E2 and tumor necrosis factor alpha cooperate to activate human dendritic cells: synergistic activation of interleukin 12 production. Journal of Experimental Medicine $186,1603-1608$.

Scandella, E., Men, Y., Gillessen, S., Forster, R., Groettrup, M., 2002. Prostaglandin E2 is a key factor for CCR7 surface expression and migration of monocyte-derived dendritic cells. Blood 100, 1354-1361.

Sheibanie, A.F., Tadmori, I., Jing, H., Vassiliou, E., Ganea, D., 2004. Prostaglandin E2 induces IL-23 production in bone marrow-derived dendritic cells. FASEB Journal $18,1318-1320$

Sheibanie, A.F., Yen, J.H., Khayrullina, T., Emig, F., Zhang, M., Tuma, R., Ganea, D., 2007. The proinflammatory effect of prostaglandin E2 in experimental inflammatory bowel disease is mediated through the IL-23 $\rightarrow$ IL-17 axis. Journal of Immunology $178,8138-8147$.

Stoy, N.S., 2002. Monocyte/macrophage initiation of organ-specific autoimmunity: the ultimate 'bystander' hypothesis? Medical Hypotheses 58, 312-326.

van Beelen, A.J., Zelinkova, Z., Taanman-Kueter, E.W., Muller, F.J., Hommes, D.W., Zaat, S.A., Kapsenberg, M.L., de Jong, E.C., 2007. Stimulation of the intracellular bacterial sensor NOD2 programs dendritic cells to promote interleukin-17 production in human memory T cells. Immunity 27, 660-669.

van de Wetering, D., de Paus, R.A., van Dissel, J.T., van de Vosse, E., 2009. Salmonella induced IL-23 and IL-1beta allow for IL-12 production by monocytes and Mphi1 through induction of IFN-gamma in CD56 NK/NK-like T cells. PLoS ONE 4, e8396.

van der Pouw Kraan, T.C., Boeije, L.C., Smeenk, R.J., Wijdenes, J., Aarden, L.A., 1995. Prostaglandin-E2 is a potent inhibitor of human interleukin 12 production. Journal of Experimental Medicine 181, 775-779.

Wang, M.T., Honn, K.V., Nie, D., 2007. Cyclooxygenases, prostanoids, and tumor progression. Cancer and Metastasis Reviews 26, 525-534. 
Wang, P., Wu, P., Siegel, M.I., Egan, R.W., Billah, M.M., 1994. IL-10 inhibits transcription of cytokine genes in human peripheral blood mononuclear cells. Journal o Immunology 153, 811-816.

Weller, C.L., Collington, S.J., Hartnell, A., Conroy, D.M., Kaise, T., Barker, J.E., Wilson, M.S., Taylor, G.W., Jose, P.J., Williams, T.J., 2007. Chemotactic action of prostaglandin E2 on mouse mast cells acting via the PGE2 receptor 3. Proceedings of the National Academy of Sciences of the United States of America 104 $11712-11717$.

Wilson, N.J., Boniface, K., Chan, J.R., McKenzie, B.S., Blumenschein, W.M., Mattson, J.D., Basham, B., Smith, K., Chen, T., Morel, F., Lecron, J.C., Kastelein, R.A., Cua, D.J., McClanahan, T.K., Bowman, E.P., de Waal Malefyt, R., 2007. Development cytokine profile and function of human interleukin 17-producing helper T cells. Nature Immunology 8, 950-957.

Yao, C., Sakata, D., Esaki, Y., Li, Y., Matsuoka, T., Kuroiwa, K., Sugimoto, Y., Narumiya, S., 2009. Prostaglandin E2-EP4 signaling promotes immune inflammation through Th1 cell differentiation and Th17 cell expansion. Nature Medicine 15, 633-640.
Yen, D., Cheung, J., Scheerens, H., Poulet, F., McClanahan, T., McKenzie, B., Kleinschek, M.A., Owyang, A., Mattson, J., Blumenschein, W., Murphy, E., Sathe, M., Cua, D.J., Kastelein, R.A., Rennick, D., 2006. IL-23 is essential for T cell-mediated colitis and promotes inflammation via IL-17 and IL-6. Journal of Clinical Investigation $116,1310-1316$

Yu, Y., Chadee, K., 1998. Prostaglandin E2 stimulates IL-8 gene expression in human colonic epithelial cells by a posttranscriptional mechanism. Journal of Immunology $161,3746-3752$.

Zhang, J., Bui, T.N., Xiang, J., Lin, A., 2006. Cyclic AMP inhibits p38 activation via CREB-induced dynein light chain. Molecular and Cellular Biology 26 , 1223-1234.

Zhang, J.S., Feng, W.G., Li, C.L., Wang, X.Y., Chang, Z.L., 2000. NF-kappa B regulates the LPS-induced expression of interleukin $12 \mathrm{p} 40$ in murine peritonea macrophages: roles of PKC, PKA, ERK, p38 MAPK, and proteasome. Cellular Immunology 204, 38-45. 